\title{
BIOMECHANICAL AND BIOCHEMICAL STUDY OF MUSCLE-TENDON-BONE IN PORCINE DIGITAL FLEXOR TENDON
}

\author{
Bryan B. Danley \\ North Carolina State University \\ Department of Mechanical \& Aerospace Engineering \\ Raleigh, North Carolina, USA \\ bbdanley@ncsu.edu
}

\author{
Hsiao-Ying Shadow Huang* \\ North Carolina State University \\ Department of Mechanical \& Aerospace Engineering \\ Raleigh, North Carolina, USA \\ hshuang@ncsu.edu
}

\begin{abstract}
The musculoskeletal system provides the body with both movement and support. In particular, contractile forces developed in the muscles are transmitted through the muscletendon junction (MTJ) into the tendon and then through the tendon-bone insertion into the bone. Each junction occurs between two dissimilar materials (muscle-to-tendon and tendon-to-bone) and neither is fully understood. The current study analyzes the relationship between the tissue microstructure and macro-scale biomechanical properties of the muscle-tendon-bone unit to better understand the anisotropic mechanical behavior of the tissue. Collagen content was assayed at various locations along the porcine digital flexor tendon. Collagen concentration as a percent of the wet weight in the bone end was found to be $20.4 \pm 5.2 \%(\mathrm{n}=6)$, the mid tendon to be $20.6 \pm 5.3 \%(\mathrm{n}=6)$, and the muscle end to be $25.2 \pm 3.6 \% \quad(n=4)$. No statistical differences were found between these collagen concentrations.

Additionally, to the best of the authors' knowledge, this is the first study to report cross-sectional stress-strain data for the muscle-tendon-bone unit. Results indicate that the tendon cross-sectional stiffness increases from the proximal end to the distal end. However, no direction dependent anisotropies were found between the mediolateral and dorsopalmar directions. Effects of microstructural components, such as glycosaminoglycans and collagen, and phenomenon such as fibril sliding and cross-linking, are discussed in relation to the reported cross-section stress-strain response. This work provides a synergistic approach for quantifying biomechanical and biochemical properties of biological tissue, and potentially facilitates the development of tissue engineered MTJ and tendon-bone insertion.
\end{abstract}

\footnotetext{
*Corresponding author
}

\section{INTRODUCTION}

The muscle-tendon-bone unit transmits contractile loads, developed in the muscle, through the tendon as a tensile load, into the bone to produce motion. The myotendinous junction (MTJ) connects the muscle to the tendon through a series of interdigitated extensions of the microstructure. The last collagen fibrils of the tendon extend into invaginations in the myofibers of the muscle and transmit loads through lateral contractions [1, 2]. The tendon-to-bone insertion is a functionally graded tissue that varies in collagen types, collagen orientation, and mineralization [3-8].

The tendon is a hierarchical tissue starting at the nanoscale with collagen molecules which aggregate into microfibrils which aggregate into fibrils which form fibers which collect to form fascicles which aggregate to form the tendon [8]. It is composed of collagen, proteoglycans, glycoproteins, water and cells [9]. The study of the muscle-tendon-bone unit is of importance because injuries of these tissues are common and they tend to heal slowly. Additionally, biomechanical properties are not restored to their original value after healing [9].

Most studies focus on the uniaxial tendon properties aligned with the primary load direction. There are few studies that consider the biaxial properties of the tendon [10, 11]. However, these studies consider one longitudinal and one transverse direction. To the best of the authors' knowledge, no previous studies have been conducted on the biomechanical properties of a cross-section of the tendon.

In this study we report stress-strain data for the tendon cross-section in various locations and relate the findings back to the biochemical composition. 


\section{MATERIAL AND METHODS}

Porcine forelimbs of large sows were obtained from the local abattoir. The skin and fatty tissue surrounding the muscletendon-bone unit was carefully cleared away from the deep digital flexor tendon of the third digit. The tendon is removed via a cut near the tendon-to-bone insertion and a cut near the muscle-tendon junction (MTJ) (tendon branches for other digits are severed near the main tendon stem).

\section{Biaxial Testing}

From each of these tendons, three cross-sections for biaxial testing were removed with two razor blades fixed together. One cross-section is extracted from the tendon proximal to the junction of flexor tendons (muscle-end, $n=8$ ), one cross-section from the middle of the third digit tendon (mid-tendon, $n=9$ ), and one cross-section from the distal end of the removed sample (bone-end) $(n=6)$. Samples that were not large enough to mount in the testing apparatus were thrown out. The average thickness of the cross-sections was $0.79 \pm 0.20 \mathrm{~mm}$. After removal from the forelimb, all cross-sections were stored in Hanks' Balanced Salt Solution (HBSS) at $4^{\circ} \mathrm{C}$ between dissection and testing.

The BioTester 5000 (CellScale Biomaterials Testing, Waterloo, ON, Canada) biaxial testing apparatus, equipped with a $0.5 \mathrm{~N}$ load cell in each axis of loading, was used to measure the force and displacement of the tissue samples. The built-in CCD camera is synchronized with the load cells and actuators and collected 1280 x 960 px images at a rate of $1 \mathrm{~Hz}$. Force and displacement data were collected at a rate of $5 \mathrm{~Hz}$ and were used to compute stress and strain data. Each tested sample was mounted to the BioTester with four BioRakes each consisting of five tungsten tines spanning $4 \mathrm{~mm}$ in width. Testing was performed in a room temperature bath of HBSS. The dorsopalmar axis of the tendon cross-section was aligned with the $\mathrm{x}$-axis of the BioTester and the mediolateral axis was aligned with the y-axis.

A pre-load of $10 \mathrm{mN}$ was applied in both axes and this state was defined as the zero strain state. Each sample was pre-

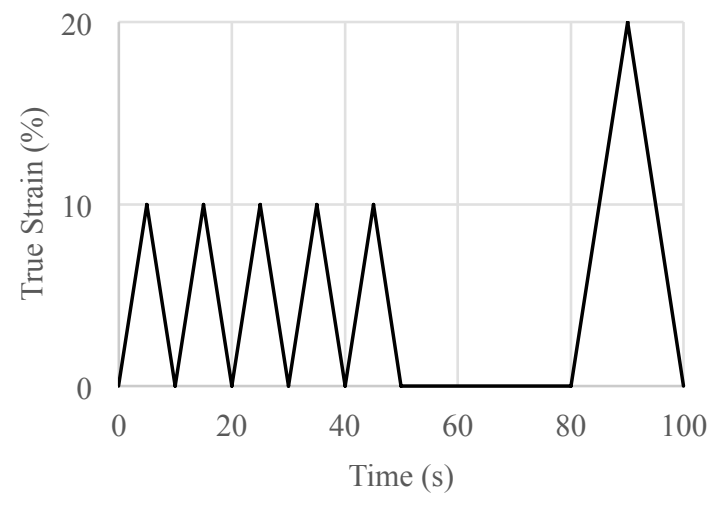

Figure 1. Biaxial testing protocol for each tendon cross-section. All stretching and recovering took place at a strain rate of $2 \% / \mathrm{s}$. conditioned equibiaxially with five successive triangular waveform to $10 \%$ true strain at a medium strain rate of $2 \% / \mathrm{s}$ [12]. After pre-conditioning samples were rested at zero strain for 30 seconds to allow the tendon to relax. This rest duration was determined during preliminary testing to allow the tendon samples to return to a zero stress state. Finally, each sample was strained equibiaxially at $2 \%$ /s to a peak of $20 \%$ true train. A summary of this testing protocol is provided in Fig. 1.

Stress and strain curves are prepared from the collected force and displacement data. These curves are broken into three strain regions: $0-7.5 \%$ (low strain), $7.5-15 \%$ (medium strain), and $15-20 \%$ (high strain). An effective elastic modulus in each strain region was computed.

\section{Collagen Assay}

A second cross-section from each of the three locations from the first six pigs were removed. From each of these 18 samples a small portion of the center was removed for use in collagen assay. Collagen concentrations (collagen types I through V) of the tendon was measured using a soluble collagen assay (Sircol; Accurate Chemical and Scientific Corp., Westbury, NY) and techniques established in our previous work [13].

Briefly, samples are weighed using an analytical balance (VWR, West Chester, PA). On average, these samples weighed $5.96 \pm 1.48 \mathrm{mg}$. A solution of $0.5 \mathrm{M}$ acetic acid and $1 \mathrm{mg} / \mathrm{mL}$ Pepsin A in distilled water is used to extract the collagen. A 50 $\mathrm{mL}$ batch of collagen extraction solution is prepared with 48.57 $\mathrm{mL}$ of distilled water and $1.43 \mathrm{~mL}$ of $17.5 \mathrm{~N}$ acetic acid (SigmaAldrich, St. Louis, MO) and $50 \mathrm{mg}$ of Pepsin A (P7000; SigmaAldrich). Each sample is extracted in $1 \mathrm{~mL}$ of the extraction solution for a period of 96 hours. Preliminary testing at various extraction times confirmed that 96 hours is sufficient for a saturated collagen extraction in porcine tendon. $100 \mu \mathrm{L}$ of the extracted collagen solution is mixed with $1 \mathrm{~mL}$ of Sirius red dye, pelleted in a centrifuge, decanted, and dissolved in $1 \mathrm{~mL}$ of alkali reagent. The absorbance of the dissolved pellets is quantified via a spectrophotometer (Thermo Fisher Scientific, Waltham, MA) at a wavelength of $550 \mathrm{~nm}$. The mass of collagen in the sample is determined by a comparison to an established collagen standard curve [13] and normalized to the wet weight.

\section{Statistical Analysis}

All data are presented as the means \pm the standard deviations. Differences in collagen concentration are tested using a single factor ANOVA. A Bonferroni multiple comparisons test with $p<0.05$ was performed to compare the elastic modulus of the tendon samples between location and direction to determine if there is a variation in biomechanical property along the length of the tendon or any anisotropy within the cross-section. All statistical analysis was conducted using GLM in SAS 9.4 (SAS Institute Inc, Cary, NC, USA). A value of $p<0.05$ was considered significant. 


\section{RESULTS AND DISCUSSION}

The biaxial testing results for the three locations (bone end, mid tendon, and muscle end) were used to produce stress versus strain curves presented in Figure 2. Representative images collected during testing are provided in Figure 3 along with point tracking results. Maximum stresses at $20 \%$ true strain for the muscle end were $35.2 \pm 34.2 \mathrm{kPa}$ in the $\mathrm{x}$-direction and $36.9 \pm 51.9 \mathrm{kPa}$ in the $\mathrm{y}$-direction, for the mid tendon were $83.8 \pm 34.4 \mathrm{kPa}$ in the $\mathrm{x}$-direction and $92.0 \pm 52.1 \mathrm{kPa}$ in the $\mathrm{y}$ direction, and for the bone end were $142.2 \pm 85.7 \mathrm{kPa}$ in the $\mathrm{x}$ direction and $110.6 \pm 77.6 \mathrm{kPa}$ in the y-direction.

From these data, effective elastic moduli were computed for each of the previously mentioned strain ranges. These results are presented in Figure 4. Using a Bonferroni multiple comparisons test with $p<0.05$, it was determined that the bone end of the tendon is stiffer in all regions of strain than the muscle end of the tendon. No differences between the bone end of the tendon and the mid tendon were detected. And the muscle end of the tendon was only more compliant in the larger strain range (15-20\%). No differences were detected between the $\mathrm{x}$ - and $\mathrm{y}$-direction stiffnesses in any strain range or location.

Results for the collagen concentration for the three locations are presented in Figure 5. Using a single factor (location) ANOVA, no differences between the groups were found.

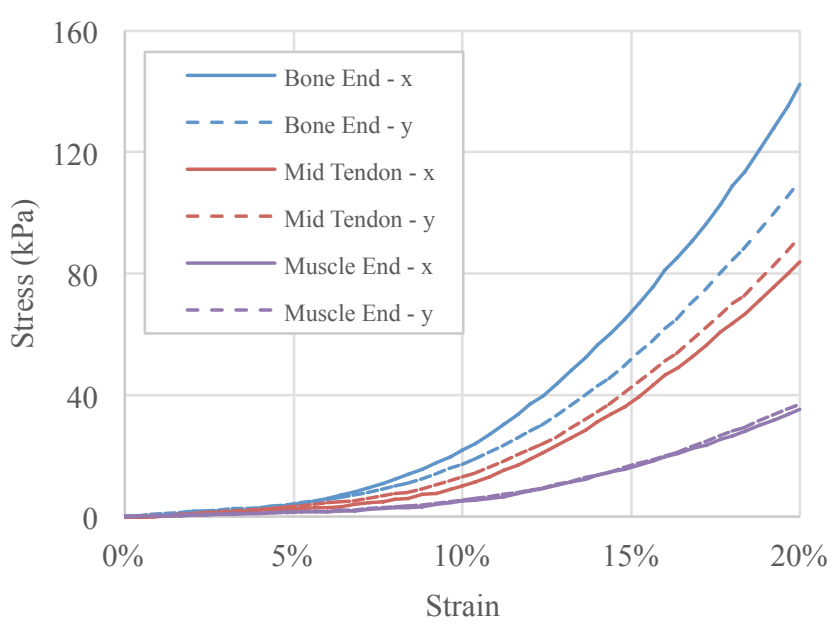

Figure 2. Average stress versus strain response for tendon crosssections collected from the bone end (blue, $n=6$ ), the mid tendon (red, $n=9$ ) and the muscle end (purple, $n=8$ ). The tendon cross-section tends to be more stiff toward the bone end and more compliant toward the muscle end. No dramatic transverse anisotropy is apparent.

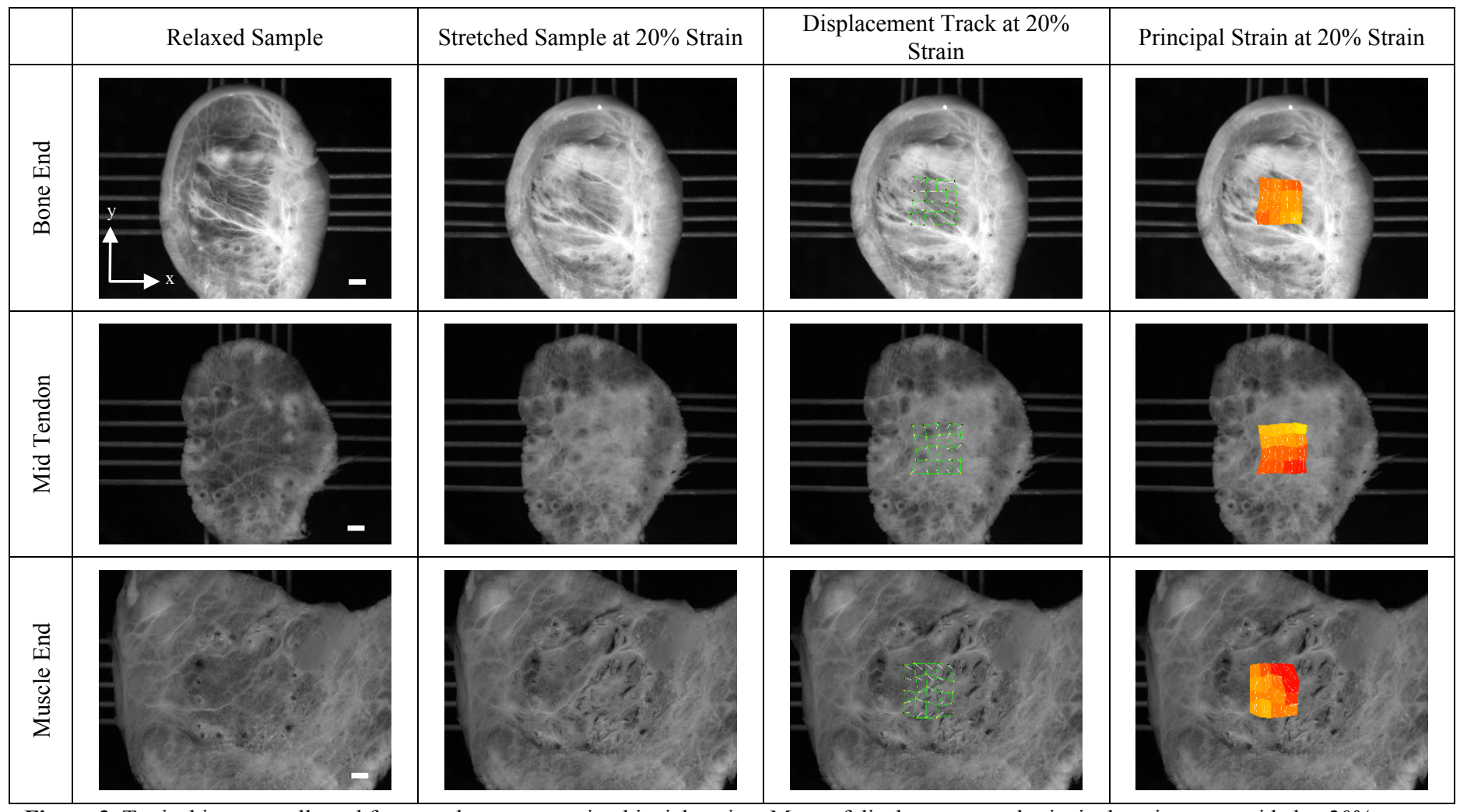

Figure 3. Typical images collected from tendon cross-section biaxial testing. Maps of displacement and principal strain are provided at $20 \%$ true strain. In strain maps, yellow corresponds to $10 \%$ strain and red corresponds to $40 \%$ strain. Scale bar represents $1 \mathrm{~mm}$; all images at the same scale. 


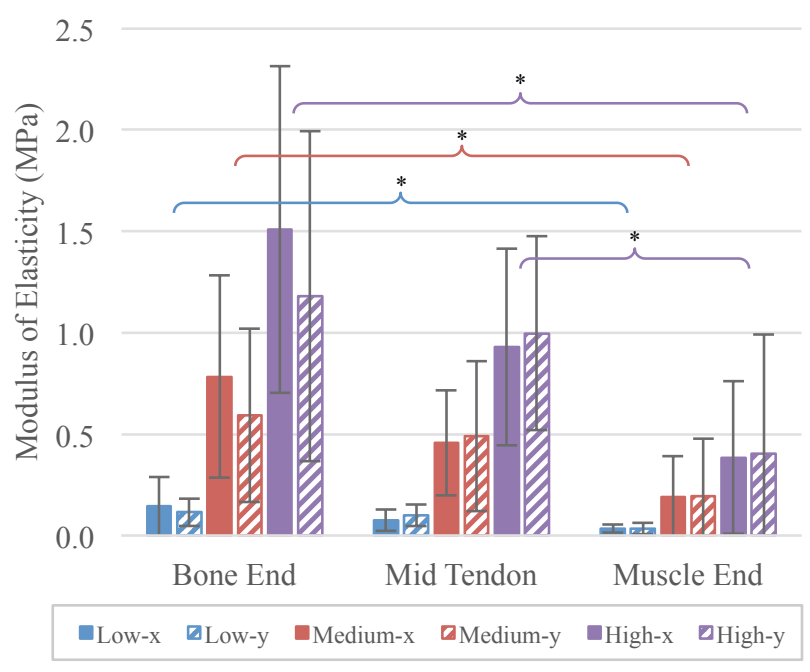

Figure 4. Modulus of elasticity for the three locations (bone end, mid tendon, and muscle end) in both the x-direction (solid) and $\mathrm{y}$ direction (diagonal) for the three strain regions: low $(0-7.5 \%$, blue), medium (7.5-15\%, red), and high (15-20\%, purple). Differences with Bonferroni correction of $p<0.05$ are denoted with an overbrace between compared values and a star.

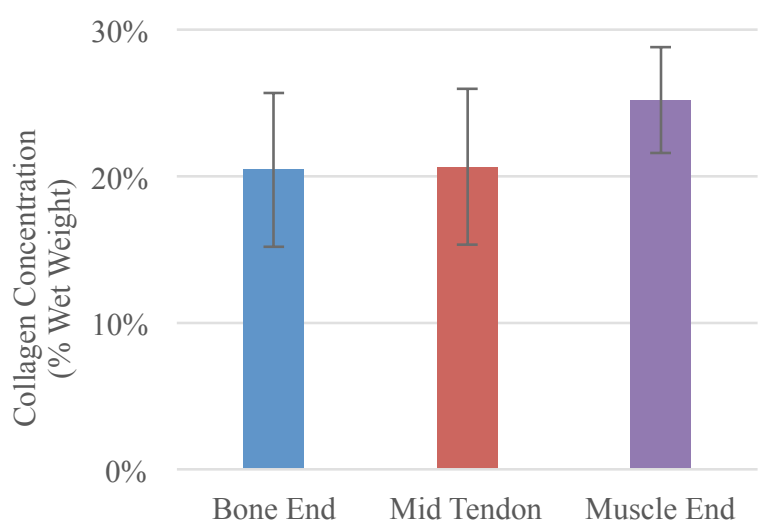

Figure 5. Collagen concentration for the bone end $(20.4 \pm 5.2 \%$, $\mathrm{n}=6)$, the mid tendon $(20.6 \pm 5.3 \%, \mathrm{n}=6)$, and the muscle end $(25.2 \pm 3.6 \%, n=4)$. No statistical differences were detected.

As with most collagenous tissue, the cross-section of the tendon (in all locations) exhibited a typical nonlinear stressstrain behavior. It is apparent in Figure 2 that the tendon stiffens along its length increasing in the direction from proximal to distal.

Because collagen fibers are not aligned in this transverse direction, it is believed that the non-collagenous ECM is responsible for the biomechanical properties. There are many studies that examine GAG cross-linking between collagen fibrils [14-16]. The primary focus of these studies are to establish a method for which loads are transmitted laterally between collagen fibrils in the context of longitudinal loading. These same cross-linking effects of the GAGs could be extended to the lateral loading studied in the current paper.
Continuing with the discussion of GAGs, it has been reported that different proteoglycans with differing GAG chains are found in different regions of the tendon based on the loading conditions (tensile or compressive) [3,17,18]. Additionally, GAG depletion in the various locations of the tendon resulted in little change in the muscle end yet weakened significantly toward the bone end [18]. This suggests that the GAG content could be cross-linked differently based on the location or more simply that different GAG content in the different regions manifest in a variation in biomechanical properties in the crosssection.

While no statistically significant differences were found between the two directions of loading, it can be seen that the bone end exhibits more apparent anisotropy than that of the mid tendon and muscle end. This can be attributed to the fact that fewer samples were tested from the bone end than the other two regions and a larger sample size would reduce the standard deviation allowing for a statistically significant difference to be detected. Furthermore, collagen orientation tends to splay closer to the tendon-to-bone insertion [3] which can lead to a more anisotropic behavior of the tissue.

Collagen concentration of the tendon has been recorded before in rabbits [19], humans [20], and avians [21]. Based on results from the rabbit tendon, tendons are $72 \%$ water and collagen constitutes $17 \%$ of the wet weight. Both the human and avian collagen concentrations are reported normalized to the dry weight. Using the water concentration from rabbit tendons, the human and avian collagen concentrations are computed to be approximately $19 \%$ and $22 \%$ of the wet weight, respectively. These results support the results collected in the present study. Because the collagen concentration was similar between three regions while different biomechanical properties were reported, we can conclude that the cross-sectional mechanical behavior is dominated by the non-collagenous ECM.

\section{CONCLUSION}

The current study, to the best of the authors' knowledge, is the first to provide stress-strain data for the cross-section of the tendon. This equibiaxial testing was performed at three locations along the porcine deep digital flexor tendon and a variation in stiffness was observed. Additionally, we report the collagen concentration in these same three regions, however, observed no variation. The result of this study will be furthered by expanding the locations and orientations tested along the muscle-tendonbone unit in an effort to improve the understanding of its unique structure and to aid in tissue engineering repair techniques.

\section{REFERENCES}

[1] Trotter J., 2002, "Structure - function considerations of muscle-tendon junctions," Comparative Biochemistry and Physiology Part A, 133 pp. 1127-1133

[2] Finni T., 2006, "Structural and functional features of human muscle-tendon unit," Journal of Applied Physiology, 98 pp. 1328-1333

[3] Thomopoulos S., Williams G., Gimbel J., Favata M., and Soslowsky L., 2003, "Variation of biomechanical, structural, 
and compositional properties along the tendon to bone insertion site," Journal of Orthopaedic Research, 21(3) pp. 413-419

[4] Thomopoulos S., Genin G., and Galatz L., 2010, "The development and morphogenesis of the tendon-to-bone insertion - what development can teach us about healing -," Journal of Musculoskeletal and Neuronal Interactions, 10(1) pp. 35-45

[5] Wang J., 2006, "Mechanobiology of tendon," Journal of Biomechanics, 39(9) pp. 1563-1582

[6] Killian M., Cavinatto L., Galatz L., and Thomopoulos S., 2012, "The role of mechanobiology in tendon healing," Journal of Shoulder and Elbow Surgery, 21(2) pp. 228-237

[7] Benjamin M., Kumai T., Milz S., Boszczyk B., Boszczyk A., and Ralphs J., 2002, "The skeletal attachment of tendons tendon 'entheses'," Comparative Biochemistry and Physiology Part A: Molecular \& Integrative Physiology, 133(4) pp. 931945

[8] Genin G., Kent A., Birman V., Wopenka B., Pasteris J., Marquez P., and Thomopoulos S., 2009, "Functional grading of mineral and collagen in the attachment of tendon to bone," Biophysical Journal, 97(4) pp. 976-985

[9] Dourte L.M., Pathmanathan L., Jawad A.F., Iozzo R.V., Mienaltowski M.J., Birk D.E., and Soslowsky L.J, 2012, "Influence of decorin on the mechanical, compositional, and structural properties of the mouse patellar tendon," Journal of Biomechanical Engineering, 134(3)

[10] Szczesny S.E., Peloquin J.M., Cortes D.H., Kadlowec J.A., Soslowsky L.J., and Elliott D.M., 2012, "Biaxial tensile testing and constitutive modeling of human supraspinatus tendon," Journal of Biomechanical Engineering, 134(2)

[11] Azizi E., and Roberts T.J., 2009, "Biaxial strain and variable stiffness in aponeuroses," Journal of Physiology, 587(17) pp. 4309-4318

[12] Gregory D.E., and Callaghan J.P., 2010, “An Examination of Strain Rate on Subfailure Mechanical Properties of the Annulus Fibrosus," Journal of Biomechanical Engineering, 132(9):091010-091010-8

[13] Huang H.Y.S, Balhouse B.N., and Huang S., 2012, "Application of simple biomechanical and biochemical tests to heart valve leaflets: Implications for heart valve characterization and tissue engineering," Proceedings of the Institution of Mechanical Engineers, Part $\mathrm{H}$ : Journal of Engineering in Medicine 226(11) pp. 868-876.

[14] Redaelli A., Vesentini S., Soncini M., Vena P., Mantero S., and Montevecchi F.M., 2003, "Possible role of decorin glycosaminoglycans in fibril to fibril force transfer in relative mature tendons - a computational study from molecular to microstructural level," Journal of Biomechanics, 36 pp. 15551569

[15] Rigozzi S., Muller R., Stemmer A., Snedeker J.G., 2012, "Tendon glycosaminoglycan proteoglycan sidechains promote collagen fibril sliding-AFM observations at the nanoscale," Journal of Biomechanics, 46(4) pp. 813-818

[16] Fessel G., and Snedeker J.G., 2011, "Equivalent stiffness after glycosaminoglycan depletion in tendon-an ultra- structural finite element model and corresponding experiments," Journal of Theoretical Biology, 268(1) pp. 77-83 [17] Matuszewski P.E., Chen Y.L., Szczesny S.E., Lake S., Elliott D.M., Soslowsky L.J., and Dodge G.R., 2012, "Regional variation in human supraspinatus tendon proteoglycans: decorin, byglycan, and aggrecan," Connective Tissue Research, 53(5) pp. 343-348

[18] Rigozzi S., Muller R., Snedeker J.G., 2009, "Local strain measurement reveals a varied regional dependence of tensile tendon mechanics on glycosaminoglycan content," Journal of Biomechanics, 42 pp. 1547-1552

[19] Taskiran D.., Taskiran E., Yercan H., and Kutay F.Z., 1999, "Quantification of total collagen in rabbit tendon by the Sirius red method," Turkish Journal of Medical Sciences, 29 pp 7-9

[20] Riley G.P., Harrall R.L., Constant C.R., Chard M.D., Cawston T.E., and Hazleman B.L., 1994, "Tendon degeneration and chronic should pain: changes in the collagen composition of the human rotator cuff tendons in rotator cuff tendinitis," Annals of the Rheumatic Diseases, 53(6) pp. 359366

[21] Tsuzaki M., Yamauchi M., and Banes A.J., 1993, “Tendon collagens: extracellular matrix composition in shear stress and tensile components of flexor tendons," Connective Tissue Research, 29(2) pp. 141-152 